\title{
EVALUACIÓN DE APRENDIZAJE SIGNIFICATIVO Y ESTILOS DE APRENDIZAJE: ALCANCES, PROPUESTA Y DESAFÍOS EN EL AULA
}

\author{
Meaningful learning assessment and learning styles: significance, \\ proposals and challenges in the classroom
}

\author{
José Salazar Ascencio \\ e-mail: jose.salazar.e@ufrontera.cl \\ Universidad de La Frontera. Chile
}

\begin{abstract}
RESUMEN
Inicialmente, se aborda el tema de la evaluación educativa a través de una serie de cuestionamientos que incluyen desde el qué evaluar hasta para quién se debe evaluar. A continuación, se desarrolla el concepto de aprendizaje significativo basado en el enfoque cognitivo de Asubel, para concluir con una propuesta de implementación de la evaluación del aprendizaje significativo en el quehacer docente, haciendo énfasis en la complejidad implícita del proceso para la recolección de evidencias de lo aprendido, así como los desafíos que conlleva la evaluación de los aprendizajes significativos, resaltando tres aspectos principales: la importancia de la construcción de instrumentos de evaluación; la integración de la autoevaluación y la coevaluación, para obtener un enfoque integral de la evaluación; y la generación de una nueva y contextualizada cultura evaluativa.
\end{abstract}

PALABRAS CLAVE: evaluación; aprendizaje significativo; instrumentos de evaluación; enfoque cognitivo; nudos.

\begin{abstract}
Initially, the issue of educational evaluation is addressed through a series of questions that include from what to evaluate to for whom to evaluate. Next, the concept of meaningful learning based on Asubel's cognitive approach is developed to conclude with a proposal for the implementation of the evaluation of meaningful learning in the teaching task, emphasizing the implicit complexity of the process for collecting evidence of the topics learned, as well as the challenges involved in evaluating meaningful learning, highlighting three main aspects: the importance of the construction of evaluation tools; the integration of self-evaluation and coevaluation, to obtain a comprehensive approach to evaluation; and the generation of a new and contextualized evaluation culture.
\end{abstract}

KEY WORDS: evaluation; meaningful learning; evaluation tools; cognitive approach; knot.

Recibido/Received: 30/07/2017

Aprobado/Aproved: 23/12/2017

Cómo referenciar este artículo / How to reference this article:

Salazar Ascencio, J. (2018). Evaluación de aprendizaje significativo y estilos de aprendizaje: alcances, propuesta y desafíos en el aula. Tendencias Pedagógicas, 31, 31-46. doi: http://dx.doi.org/10.15366/tp2018.31.001 
"El aprendizaje significativo es muy importante en el proceso educativo porque es el mecanismo humano por excelencia para adquirir y almacenar la vasta cantidad de ideas e información representadas por cualquier campo del conocimiento" (Ausubel, 1976, p. 78)

\section{INTRODUCCIÓN}

Se puede afirmar que el concepto de evaluación como objeto de estudio pedagógico, concebido por Ralph Tyler (1942), quien recoge este concepto desde el mundo industrial, para acuñar lo que se conoce como evaluación educativa. En los siguientes cuarenta años, se generó una especialización en lo referente a la evaluación de los aprendizajes, dando una permanente prioridad por su inmediatez en el aula, situación que se ha visto reflejada en el ámbito de la literatura pedagógica, a través de la apertura de un espacio de constantes investigaciones, que se visibilizan no sólo en los múltiples libros y artículos que se han publicado al respecto, sino además, en los constantes eventos que convoca la comunidad científica, tales como; congresos, simposios, seminarios, coloquios, entre otros; espacios donde el conocimiento se transforma en plataforma de productividad que, profundiza, orienta y reformula, al quehacer docente.

Tal como lo expone Salazar (2009), antes de poner en marcha cualquier proceso evaluativo en ambiente escolar, es menester tener respuestas concretas y públicas a los cuestionamientos que determinan la calidad del mismo. Por tal razón, es preciso explorar lo que todo docente y estudiante debe tener identificado en lo que se denomina "contrato evaluativo", más aún si se trata de evidenciar el nivel y logro de los aprendizajes por adquirir.

El primer cuestionamiento se relaciona con el tema, ¿QUÉ EVALUAR? Aunque parezca obvio, su respuesta es el aprendizaje del discente, sí pero es aquí donde empiezan las dificultades. ¿De qué tipo de aprendizaje estamos hablando?, es decir, ¿Qué es lo que se desea evaluar?: Habilidades, Destrezas, Capacidades o Competencias (Román. 1999), no cabe duda que éstas no son sinónimos, sino que se expresan de manera progresiva y jerárquica. Más allá de lo que explicitan los planes y programas de estudios, sobre el tipo de aprendizaje que se ha de promover en los educandos, es importante que el quehacer docente permita un tratamiento adecuado para cada aprendizaje, que sea coherente con su evaluación.

Por su parte, el cuestionamiento ¿A QUIÉN EVALUAR?, apunta de manera exclusiva a quién se debe demostrar que aprendió, es decir, el discente. Pero el proceso instruccional por su naturaleza biunívoca, entrega antecedentes que permite realizar extrapolaciones, cuya información es dable utilizarla como referencial en aspectos de la evaluación docente (Salazar 2013a).

A su vez, el tercer cuestionamiento ¿PARA QUÉ EVALUAR?, define el sentido de la evaluación, que puede ser para: seleccionar, medir, calificar, planificar, sancionar, etc. Desde hace unas décadas, las tendencias en el 
mundo pedagógico, hacen hincapié en una evaluación que apunta a la mejora, situación que exige sin duda, una mirada renovada de la evaluación, que obliga necesariamente a pensar en establecer una nueva cultura evaluativa en los centros escolares, que supere con creces las funciones administrativas centradas en el registro de calificaciones. En este sentido, la idea es que el proceso evaluativo se transforme en sí en una retroalimentación permanente, que permita efectivamente la mejora de los aprendizajes del discente.

En este contexto surge entonces, la pregunta ¿QUIÉN EVALÚA?, sin duda que un proceso tan complejo como gravitante, como lo es el aprendizaje y su evaluación, no sólo la heteroevaluación es suficiente, es posible retomar la recomendación formulada por Salazar (2007), quien sostiene que es preciso incorporar el máximo número de informantes, que puedan aportar elementos para juzgar de forma adecuada el aprendizaje. Por tal razón, cobra relevancia las instancias de: autoevaluación y coevaluación, como también la necesaria y siempre útil opinión de los padres o tutores (Salazar, Isla y Muñoz, 2002). De esta manera, ... los antecedentes proporcionados por estos informantes durante el proceso en cuestión, no pueden analizarse de manera aislada, muy por el contrario, la idea es generar una instancia de integración, donde la triangulación o cuadroangulación son las técnicas que garantizan dicha condición (Salazar, 2013a, p.173).

Si se asume que la evaluación debe cumplir la función de mejorar el aprendizaje de los estudiantes, entonces, ante el cuestionamiento ¿CÓMO EVALUAR?, surge como condición la innovación evaluativa, que permite garantizar un proceso auténtico, alternativo y de desempeño de la misma. Es en este punto donde las Tablas de Desempeño y sus correspondientes rúbricas, deben dar cuenta de la evolución del aprendizaje (Salazar e Isla. 2003). De esta manera, múltiples son los instrumentos que brindan absoluta seguridad a la hora de recoger la información requerida, entre los cuales podemos mencionar: Portafolio, Diario de clases, Entrevistas, Cuestionarios, etc. (Salazar, 2013b, p. 91).

El sexto cuestionamiento corresponde al ¿CUÁNDO EVALUAR?, lejos quedan las propuestas de fragmentar la evaluación en determinados momentos (inicio, durante y final). En este sentido, cobra relevancia los aportes de Ahumada (2001), quien señala que "la evaluación es un proceso y no un suceso". En esta dirección, se habrá de apostar por una evaluación cíclica y permanente, que esté durante todo el proceso instruccional... de manera de corregir in-situ cualquier desviación de éste, rentabilizando los tiempos y haciendo de la eficacia en el logro del aprendizaje una realidad (Salazar, 2013a, p. 174).

Tal vez la última pregunta que se ha de considerar es: ¿PARA QUIÉN EVALUAMOS?, su respuesta tiene aristas eminentemente políticas, ya que la decisión puede ir desde evaluar los aprendizajes para los intereses de la institución en la que labora el docente, hasta evaluar para la sociedad, de manera que esta juzgue la calidad de lo aprendido, pasando sin duda, por evaluar los aprendizajes al servicio del sistema educativo, donde en muchos países quedan supeditados a la presencia permanente de pruebas estandarizadas, las que se han transformados en indicadores de calidad educativa. 
El apostar por una evaluación de aprendizajes que atienda los requerimientos educativos en una sociedad del conocimiento, exige que preliminarmente la diada docente - discente reflexione sobre los cuestionamientos antes señalados. De esta manera, es urgente instalar una nueva cultura evaluativa, cuya materialización en las aulas, precisa de una reingeniería del claustro de profesores, donde se potencie la reflexión pedagógica, por sobre los habituales discursos administrativos. Por último, es menester que en la formación inicial docente, las universidades garanticen desarrollar competencias evaluativas donde el futuro profesor, demuestre un tratamiento focalizado en aprendizajes significativos, en el marco de una evaluación auténtica, alternativa y de desempeño.

\section{EL APRENDIZAJE SIGNIFICATIVO}

\subsection{Desde la semántica a sus particularidades}

El aprendizaje significativo surge en la década de los sesenta, como un aporte que realiza David Ausubel (1963 y 1968) cuando propone la teoría del aprendizaje verbal significativo. Aunque debemos reconocer que, en los espacios educacionales, este concepto adquiere importancia en la década de los noventa, momento en que se apuesta por una enseñanza basada en el paradigma constructivista, el cual debe promover el cambio conceptual y facilitar el aprendizaje significativo (Moreira, 2000). En esta dirección, la idea sublime sobre el impacto para la enseñanza y el aprendizaje, se puede sintetizar en la siguiente expresión: "Si tuviese que reducir toda la psicología educativa a un solo principio, diría lo siguiente: el factor aislado más importante que influye en el aprendizaje, es aquello que el aprendiz ya sabe. Averígüese esto y enséñese de acuerdo a ello." (Ausubel, Novak y Hanesian, 1991, p. 42).

En este sentido, diversos autores han realizado del aprendizaje significativo su objeto de estudio, del cual destacamos las siguientes definiciones:

"... proceso de elaboración de una representación personal sobre un objeto de la realidad o contenido que pretendemos aprender, por medio del cual modificamos lo que ya poseíamos e interpretamos lo nuevo de forma peculiar, para poder integrarlo y hacerlo propio, otorgándole significado" (Solé y Coll, 1993, p.16).

"Proceso a través del cual una misma información se relaciona, de manera no arbitraria (no literal), con un aspecto relevante de la estructura cognitiva del individuo." (Moreira, 2000, pp. 10-11).

“... es aquel que conduce a la creación de estructuras de conocimiento mediante la relación sustantiva entre la nueva información y las ideas previas de los estudiantes" (Díaz - Barriga y Hernández, 2005, p. 39).

Sin duda alguna, estamos ante un aprendizaje cuya naturaleza fundamental es la estructura cognitiva, en donde la preexistencia de éste influirá y facilitará el aprendizaje subsecuente, siempre que su contenido haya sido aprendido significativamente. En el siguiente cuadro (№ 1), se establece un resumen sobre las características de este enfoque cognitivo. 
Evaluación de aprendizaje significativo y estilos de aprendizaje: alcances, propuesta y desafíos en el aula

Cuadro No 1: "Enfoque cognitivo de Ausubel, pilar del paradigma constructivista del aprendizaje" (Díaz - Barriga y Hernández, 2005, p.31)

\begin{tabular}{|c|c|c|c|c|}
\hline \multirow[t]{2}{*}{ Concepción } & \multicolumn{4}{|c|}{ Metáfora educativa } \\
\hline & Alumno & Profesor & Enseñanza & Aprendizaje \\
\hline $\begin{array}{l}\text { Desarrollo } \\
\text { del } \\
\text { pensamiento } \\
\text {, aprendizaje } \\
\text { significativo y } \\
\text { resolución de } \\
\text { problemas }\end{array}$ & $\begin{array}{l}\text { Procesador } \\
\text { activo de la } \\
\text { información }\end{array}$ & $\begin{array}{lr}\text { Organizador de la } \\
\text { información } & \\
\text { tendiendo } & \\
\text { puentes } & \\
\text { cognitivos, } & \\
\text { promotor } & \text { de } \\
\text { habilidades } & \text { del } \\
\text { pensamiento } & \text { y } \\
\text { aprendizaje } & \end{array}$ & $\begin{array}{lr}\text { Inducción } & \text { de } \\
\text { conocimiento } & \\
\text { esquemático } \\
\text { significativo y de } \\
\text { estrategias } \\
\text { habilidades } \\
\text { cognitivas cómo } \\
\text { del aprendizaje }\end{array}$ & $\begin{array}{l}\text { Determinado } \\
\text { por } \\
\text { conocimiento } \\
\text { s o } \\
\text { experiencias } \\
\text { previas }\end{array}$ \\
\hline
\end{tabular}

Al revisar la literatura existente, nos encontramos que Ausubel et al. (1991), distingue tres tipos de aprendizaje significativo,: representacional, de conceptos y proposicional. El primero de ellos (representacional), es el más elemental de los aprendizajes significativos, de él dependen los otros dos restantes. "Supone la atribución de significados a determinados símbolos (típicamente palabras) ... Una determinada palabra (u otro símbolo cualquiera) representa o es equivalente en significado, determinados referentes, es decir, significa la misma cosa” (Moreira. 2000, p. 20). Por su parte, el aprendizaje de conceptos, se caracteriza por que los conceptos son representados por símbolos particulares, pero son genéricos o categóricos, ya que representan regularidades en objetos o eventos, los que son definidos por Ausubel, et al., (1991, p.86) como: "objetos, eventos, situaciones o propiedades que poseen atributos criteriales comunes y se designan, en una cultura dada, por algún signo o símbolo aceptado". Por último, el aprendizaje proposicional, no apunta a aprender significativamente lo que representan las palabras aisladas 0 combinadas, como ocurre en el aprendizaje representacional, sino que, se trata de aprender el significado de las ideas en forma de proposición, es decir, se ha de "aprender el significado que está más allá de la suma de los significados de las palabras o conceptos que componen la proposición” (Moreira, 2000, p. 22).

Con el ánimo de visibilizar el concepto de aprendizaje significativo en toda su extensión, es que se presenta la siguiente Figura (№1), en él se puede observar un mapa conceptual, que da cuenta de las características esenciales de este concepto. 
Figura № 1: Concepto de aprendizaje significativo (Ausubel,

1976; Novak y Gowin, 198B; Ontoria, 1993. Citado por Diaz - Barriga y Hernández. 2005: 44]

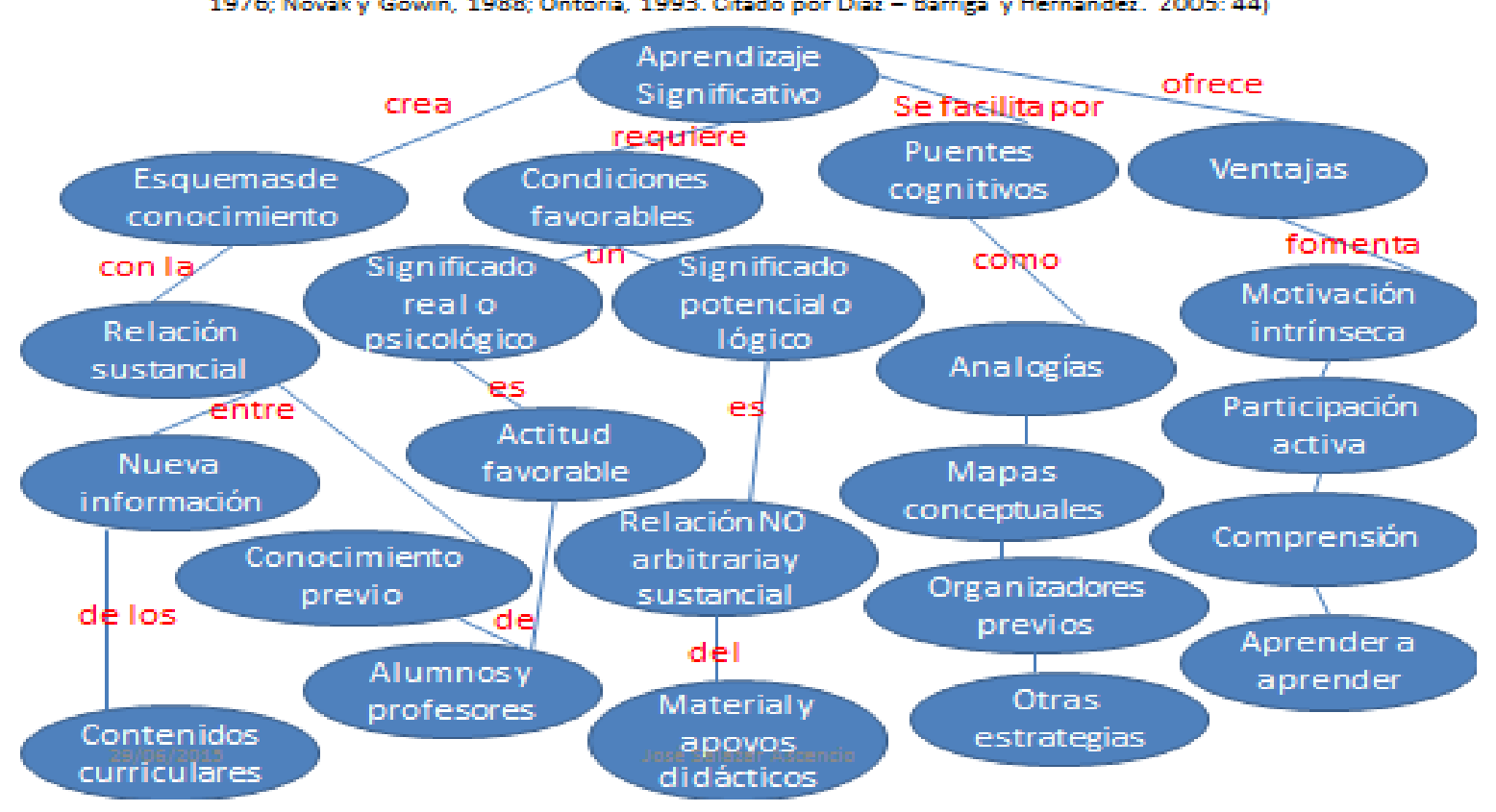

En la educación formal, es el aula el espacio preferencial donde se forjan los aprendizajes escolares. Por tal razón, el promover aprendizajes significativos exige un tratamiento en el que se debe cautelar no sólo el diseño de planificación curricular, sino el quehacer docente frente a los estudiantes. Por tal razón, es importante retomar la recomendación que hace Shuell (1990) respecto a las fases que ha de tener el aprendizaje significativo en el aula, tomando como referencia los momentos de inicio, desarrollo y cierre de una clase. Lo antes señalado queda del todo resumido en el siguiente Cuadro (№2). 


\section{Cuadro № 2: Fases del aprendizaje significativo en el}

aula (Shuell, 1990. Citado por Díaz-Barriæa y Hernández, 2005: 46))

\begin{tabular}{|c|c|c|}
\hline Inicial & Desarrollo & Cierre \\
\hline $\begin{array}{l}\text { Hechoso partes de } \\
\text { información que están } \\
\text { aislados conceptualmente }\end{array}$ & $\begin{array}{l}\text { Formación de estructuras a partir } \\
\text { de las partes de información } \\
\text { aisladas }\end{array}$ & $\begin{array}{l}\text { Mayor integración de } \\
\text { estructurasy } \\
\text { esquemas }\end{array}$ \\
\hline $\begin{array}{l}\text { Memoriza hechosy usa } \\
\text { esquemaspreexistentes }\end{array}$ & $\begin{array}{l}\text { Compresión más profunda de los } \\
\text { contenidos por aplicarlos a } \\
\text { situaciones diversas }\end{array}$ & $\begin{array}{l}\text { Mayor control } \\
\text { automático de } \\
\text { situaciones }\end{array}$ \\
\hline El procesamiento es global & $\begin{array}{l}\text { Hay oportunidad para la reflexióny } \\
\text { recepción de realimentación sobre } \\
\text { la ejecución }\end{array}$ & $\begin{array}{l}\text { Ejecución automática, } \\
\text { inconscienteysin } \\
\text { tanto esfuerzo }\end{array}$ \\
\hline $\begin{array}{l}\text { La información adquirida es } \\
\text { concretay vinculada al } \\
\text { contexto específico }\end{array}$ & $\begin{array}{l}\text { Conocimiento más abstractoy } \\
\text { puede ser generalizado }\end{array}$ & \multirow{3}{*}{$\begin{array}{l}\text { Aprendizaje basado en } \\
\text { la: a) acumulación de } \\
\text { nuevos hechos } \\
\text { (dominio) } \\
\text { preexistentesy b) } \\
\text { Interrelación de los } \\
\text { elementos de las } \\
\text { estructuras (esquemas) }\end{array}$} \\
\hline $\begin{array}{l}\text { Ocurre en formassimples de } \\
\text { aprendizaje }\end{array}$ & Uso de estrategiasmás sofisticadas & \\
\hline $\begin{array}{l}\text { Gradualmenteseva } \\
\text { formando una visión } \\
\text { globalizadora del dominio }\end{array}$ & Organización y Mapeo Cognitivo & \\
\hline
\end{tabular}

\subsection{La evaluación del aprendizaje significativo}

Una de las grandes complejidades que experimenta la evaluación en nuestros días, es el recoger evidencias de lo aprendido. Esto adquiere mayor relevancia, cuando se focaliza la evaluación en aprendizajes significativos, debido a que lo verificable es la comprensión genuina de un concepto o proposición, lo que exige estar en posesión de significados claros, precisos, diferenciados y transferibles. Surge entonces, la imperiosa necesidad que las prácticas evaluativas - en concordancia con la planificación curricular de aula-, centren su prioridad en los conocimientos de tipo conceptual y procedimental (Salazar y Marques, 2012).

Múltiples son las recomendaciones que nos entrega la literatura, referida a abordar la evaluación desde la perspectiva psicoeducativa y/o constructivista, al respecto este proceso, ha sido objeto preferencial de estudios, con temáticas tales como: los procesos de aprendizaje (Marchesi y Martín, 1998); la significatividad de los mismos (Coll y Martín, 1993); la funcionalidad (Marchesi y Martín, 1996); la contextualización (Díaz - Barriga y Hernández, 2005); la autoevaluación y la autorregulación (Salazar, 2012; Castillo y Cabrerizo, 2003a); la coherencia (Salazar e Isla, 2003); las técnicas (Barberá, 1999; Bonvecchio y Maggioni, 2005); los instrumentos (Castillo y Cabrerizo, 2003b); los procedimientos (Alfageme y Millares, 2009); etc.

A modo de síntesis de los tres últimos criterios antes señalados, en el siguiente cuadro (№ 3) se presenta un resumen que sintetiza las técnicas, procedimientos e instrumentos de evaluación de aprendizaje. 
Cuadro No 3: "Técnicas, Procedimientos e Instrumentos de Evaluación de Aprendizajes" (Salazar. 2013b, pp. 92 y 93)

\begin{tabular}{|c|c|c|}
\hline Técnicas & Procedimientos & Instrumentos \\
\hline \multirow{9}{*}{ Observacionales } & \multirow{6}{*}{ Sistemática } & Lista de Cotejo \\
\hline & & Escala de Apreciación \\
\hline & & Autoinformes \\
\hline & & Cuestionarios \\
\hline & & Diario de Clases \\
\hline & & Libreta de Clases \\
\hline & \multirow{3}{*}{ No Sistemática } & Autobiografía \\
\hline & & Sociograma \\
\hline & & Anecdotarios \\
\hline \multirow{27}{*}{$\begin{array}{l}\text { Producción de los } \\
\text { Estudiantes }\end{array}$} & \multirow{5}{*}{ Oral } & Interrogación \\
\hline & & Disertaciones \\
\hline & & Representaciones \\
\hline & & Debates \\
\hline & & Uso de Analogías \\
\hline & \multirow{12}{*}{ Escrito } & Elección Múltiple \\
\hline & & Verdadero Falso \\
\hline & & Términos Pareados \\
\hline & & Respuesta Breve \\
\hline & & Respuesta Combinada \\
\hline & & Desarrollo \\
\hline & & Informes \\
\hline & & Monografías \\
\hline & & Paper \\
\hline & & Asociación Libre y Relaciones \\
\hline & & Ponerse en el lugar de ... \\
\hline & & Generación de Preguntas \\
\hline & \multirow{5}{*}{ Representaciones Gráficas } & Mapas Conceptuales \\
\hline & & Modelos \\
\hline & & Maquetas \\
\hline & & Planos \\
\hline & & Dibujos e Imágenes \\
\hline & \multirow{5}{*}{ Prácticas } & Portafolio \\
\hline & & Proyecto \\
\hline & & Seminario \\
\hline & & Situacionales o Simulación \\
\hline & & Método de Casos \\
\hline \multirow{4}{*}{$\begin{array}{l}\text { De } \\
\text { Intercomunicación }\end{array}$} & \multirow[t]{2}{*}{ Face to Face } & Entrevista \\
\hline & & Microentrevista \\
\hline & \multirow[t]{2}{*}{ Grupal } & Coloquio \\
\hline & & Focus Group \\
\hline
\end{tabular}

El gran desafío que presenta la evaluación de cara a recoger evidencias de aprendizaje, es la necesidad de garantizar su coherencia, que se constituye en uno de los denominados nudos de la evaluación educativa. Esto es sin duda, lo más complejo en la práctica docente, debido a que se debe armonizar; qué debe aprender el alumno (objetivo), de qué manera va a ejercitar dicho aprendizaje (actividad de aprendizaje), y cómo se va a evidenciar lo aprendido 
(evaluación). La literatura nos indica que la perfecta congruencia en esta triangulación (aprendizaje - actividad y evaluación), determina la efectividad del aprendizaje (Ahumada, 2005 y Ministerio de Educación, 2006). Por su parte, las crecientes investigaciones en materia de aprendizajes, exige integrar un cuarto elemento, este dice relación con los Estilos de Aprendizaje (Alonso, Gallego y Honney, 2007), es decir, se debe considerar la manera en que aprende el estudiante. De esta manera, habremos pasado a una cuadroangulación de elementos, donde se ha de comprender que la evaluación es efectivamente un elemento esencial del curriculum y no un apéndice de éste, ya que sólo así, será posible integrar las prácticas evaluativas en el desarrollo de los aprendizajes. (Salazar, 2012).

Al buscar el sustrato que evidencie la coherencia de los aprendizajes y su evaluación, nos habremos de remitir a los aportes que proporciona Ahumada (2001 y 2005), quien esquematiza mediante el cubo de congruencia evaluativa (Ver figura № 2), la relación entre la triada: conocimientos; intencionalidad y evaluación. Si bien es cierto, dicho autor categoriza los aprendizajes en cinco, dos de ellos (actitudinal y estratégicos) tiene la connotación de ser transversales. A su vez, el proceso evaluativo lo focaliza en tres, centrándolo en los conocimientos previos, los procesos y los resultados. Es esta figura que explicita gráficamente los tres tipos de aprendizajes significativos propuestos por Ausubel, es decir, los aprendizajes representacionales -o factuales-, que se caracterizan porque su intencionalidad es reproductiva o memorística(basados en la atribución de significado a determinadas palabras, las que puedan ser nombres, lugares, fechas, etc.); los aprendizajes conceptuales, cuya característica es comprensiva (los conceptos son representados por símbolos particulares -genéricos o categóricos-, ya que materializan regularidades en objetos o eventos); y por último, los aprendizajes proposicionales -o procedimental-, cuya intencionalidad es eminentemente transferencial. 
Figura № 2: Cubo de congruencia evaluativa (Ahumada. 2001, p. 54 y 2005, p. 61)

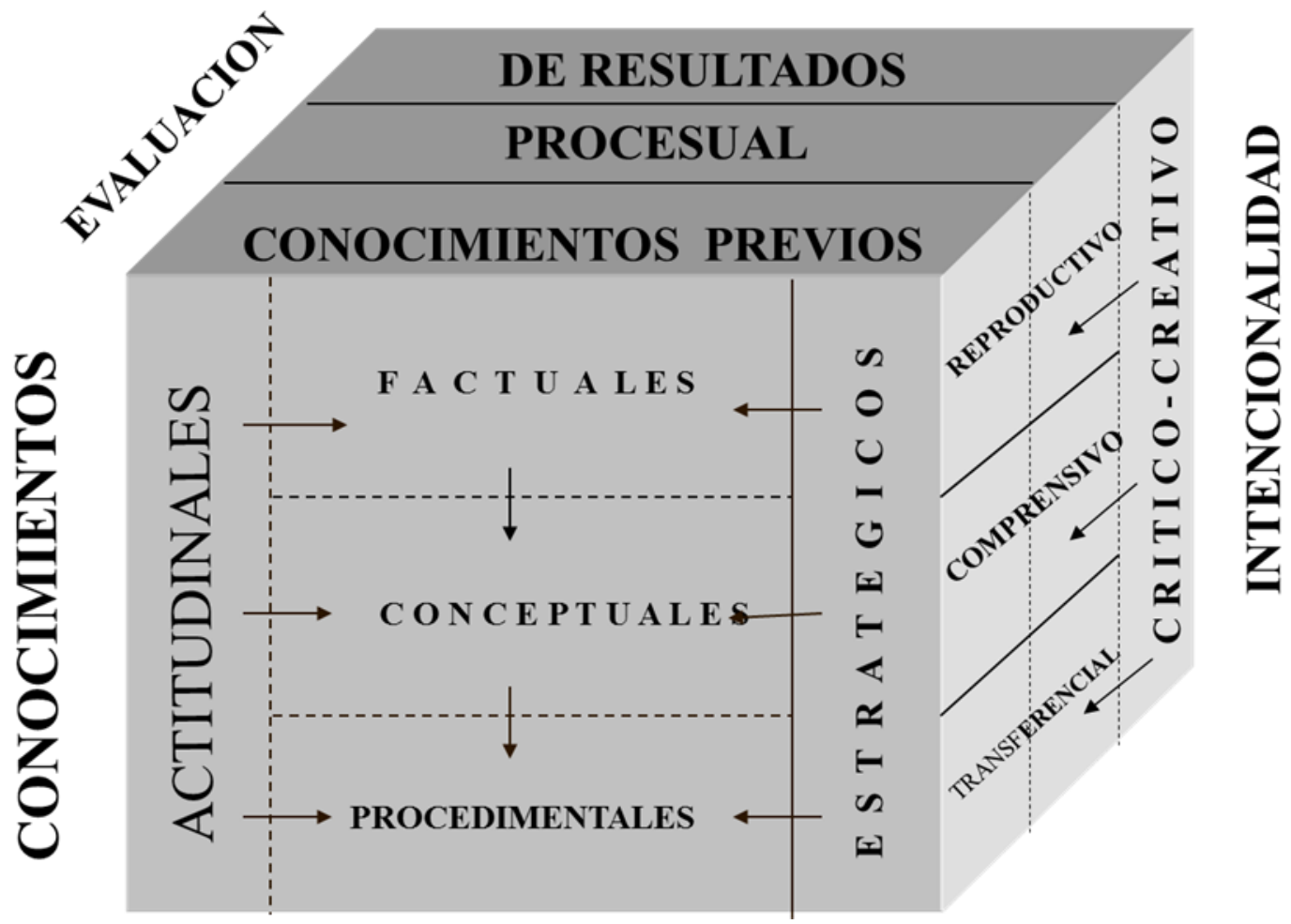

\subsection{Propuesta para su implementación en el quehacer docente}

Antes de formular una propuesta de implementación para evaluar aprendizajes significativos, es necesario señalar que al menos, se precisan dos requerimientos. El primero de ellos, dice relación con garantizar efectivamente en el diseño curricular, tanto en su planificación como en su ejecución en el aula, la promoción efectiva de dichos tipos de aprendizaje. En este sentido, la recomendación tiene una doble orientación, por un lado, apostar por la metacognición, dando luz verde a los aprendizajes conceptuales y proposicionales, y por otro lado, cautelar la intencionalidad, comprensiva y transferencial respectivamente. El segundo requerimiento, apunta a establecer ambientes de aprendizaje que permitan en los educandos, desarrollar el interés por hacer del aprendizaje un espacio de desafío permanente. Asimismo, el focalizar la enseñanza considerando las mejores condiciones en el aula, y respetando los estilos de aprendizaje, no sólo permite asegurar el abordaje adecuado de los aprendizajes significativos, sino que además, garantiza el tan anhelado tratamiento de la diversidad.

La evaluación de los aprendizajes significativos, tiene su motor en la elaboración de los instrumentos que permiten recoger las evidencias de los mismos, las que sin dudas deben ser concordantes con su intencionalidad. De esta manera, el Cuadro (№ 4) siguiente permite diseñar la evaluación, de manera tal que se pueda cautelar la necesaria e imprescindible coherencia evaluativa. La matriz en cuestión es una herramienta, que todo docente puede emplear, con el objeto de organizar e implementar su propuesta de evaluación de aprendizajes. 
Seleccionada la asignatura, unidad y curso, se ha de descomponer el (los) objetivo(s) general(es) (A) que propone la unidad, en objetivos de aprendizaje (A1, A2, A3..., An), es decir, la suma de estos últimos cubre a plenitud el propósito del (de los) aprendizaje(s) definido(s) en la unidad curricular. En este ejercicio, para cada objetivo de aprendizaje se ha de declarar su naturaleza $\left({ }^{*}\right)$ (Factual $(F)$, Conceptual $(C)$, Procedimental $(P)$, Actitudinal $(A)$, o Estratégico $(E))$ y su correspondiente intencionalidad $\left({ }^{* *}\right)$, ya sea Reproductivo (R), Comprensivo (C), Transferencial (T), Valórico (V) o Estrategias de Aprendizaje (EA).

El paso siguiente consiste en que, una vez identificados los aprendizajes a evaluar (A1, A2, A3...,An), es necesario señalar los instrumentos con los cuales se recogerá la evidencia. Por lo tanto, cobra significado el Cuadro № 3 expuesto anteriormente, en el que se mencionan 40 instrumentos, de los cuales el docente puede seleccionar el más adecuado, dependiendo de la naturaleza de lo que se quiere evaluar y la experiencia evaluativa que tiene en el quehacer docente. En el marco del diseño de la evaluación, es probable que un mismo instrumento pueda ser empleado en más de un aprendizaje (A1 y A3), o al revés, en un aprendizaje sea posible utilizar más de un instrumento (A2).

Es preciso que para cada instrumento se construyan sus correspondientes ítems, cautelando la coherencia entre la naturaleza del objetivo con el(los) ítem(s) construido(s), es decir, si el objetivo tiene como naturaleza la comprensión, el(los) ítem(s) tiene(n) que permitir recoger la evidencia comprensiva, de la misma manera ocurre en las diadas: factual reproductivo; procedimental - transferencial; actitudinal - valórico; y estratégico - estrategias de aprendizaje.

El peso específico de los ítems (Pé), determina el reconocimiento de la relevancia de los aprendizajes a evaluar de cara a una eventual calificación, pudiendo ser expresados en porcentajes o puntuaciones. La precaución que hace debe tener, es garantizar una relación de proporcionalidad basándose en la complejidad del aprendizaje, es decir, los aprendizajes factuales al ser reproductivos, deben tener menor peso que los conceptuales que son comprensivos, y éstos a su vez deben ser menores que los procedimentales que son transferenciales. Con respecto a los aprendizajes actitudinales y estratégicos, al tratarse de estructuras transversales del curriculum, sin duda que se han de evaluar, pero se sugiere que no se les califique, es por ello, que en la matriz de congruencia no se les asigna porcentaje o puntuación alguna. Por último, en la dinámica del proceso evaluativo está la posibilidad de definir el grado de participación de los estudiantes $\left.{ }^{* * *}\right)$, ya sea individual (I) o grupal $(\mathrm{G})$, que dependerá del contrato evaluativo vigente, el que será socializado al inicio de la unidad curricular.

Cuadro № 4: Matriz de congruencia para la elaboración de instrumentos de evaluación

Asignatura:

Unidad: 
Curso:

\begin{tabular}{|c|c|c|c|c|c|c|c|c|}
\hline \multirow{2}{*}{$\begin{array}{l}\text { Objetivo } \\
\text { general }\end{array}$} & \multicolumn{3}{|c|}{$\begin{array}{l}\text { Objetivo de } \\
\text { aprendizaje }\end{array}$} & \multirow{2}{*}{ Instrumento } & \multirow{2}{*}{ Ítem } & \multirow{2}{*}{ Pé } & \multicolumn{2}{|c|}{$\star \star \star *$} \\
\hline & $\begin{array}{c}\text { Enunciad } \\
0\end{array}$ & * & $\star *$ & & & & I & G \\
\hline \multirow{8}{*}{$A$} & A1 & $F$ & $\mathrm{R}$ & Elección Múltiple & 1,2 y 3 & 10 & $X$ & \\
\hline & $\mathrm{A} 2$ & C & C & $\begin{array}{c}\text { Debate / } \\
\text { Disertación }\end{array}$ & $\begin{array}{c}\text { Rubrica 1/ } \\
2\end{array}$ & 15 & & $X$ \\
\hline & A3 & $\mathrm{C}$ & $\mathrm{C}$ & Elección Múltiple & 4 y 5 & 15 & $\mathrm{X}$ & \\
\hline & A4 & $P$ & $\mathrm{~T}$ & Monografías & Rubrica 3 & 30 & $\mathrm{X}$ & \\
\hline & A5 & $P$ & $\mathrm{~T}$ & Desarrollo & Rubrica 4 & 30 & $X$ & \\
\hline & A6 & A & V & Lista de Cotejo & $1,2,3$ y 4 & - & $\bar{X}$ & \\
\hline & A7 & $E$ & EA & Lista de Cotejo & $5,6,7$ y 8 & - & & $X$ \\
\hline & $A n$ & & & & & & & \\
\hline
\end{tabular}

*= Naturaleza: Factual (F); Conceptual (C); Procedimental (P); Actitudinal (A) 0 Estratégico $(\mathrm{E})$

${ }^{* *}=$ Intencionalidad: Reproductivo (R); Comprensivo (C); Transferencial (T); Valórico (V) o Estrategias de Aprendizaje (EA).

Pé= Peso Específico del (de los) ítem(s).

${ }^{* * *}=$ Grado de Participación: I= Individual; G= Grupal

\section{COMENTARIOS FINALES: LOS DESAFÍOS DE LA EVALUACIÓN EN LOS APRENDIZAJES SIGNIFICATIVOS}

En materia de evaluación de los aprendizajes, una sentida necesidad docente es solicitar capacitación en construcción de instrumentos de evaluación, pensando que esta competencia pedagógica no es adquirida en la formación inicial de los maestros, o en su defecto, no hay claridad para enfrentar este proceso, con una mirada basada en la innovación. Más allá del grado de veracidad al respecto y que moviliza a muchos centros escolares, en la actualización de sus profesores(as) en materias profesionales, es necesario señalar que la construcción de instrumentos, no garantiza que las instituciones educativas cumplan con su función prioritaria, que consiste en que el estudiante adquiera los aprendizajes que contempla el curriculum. Por tal razón, es necesario potenciar en el quehacer del aula, competencias que evidencien su relación con la capacidad de un tratamiento adecuado a los aprendizajes curriculares, para ello es fundamental saber promover el aprendizaje significativo.

Es importante señalar que la propuesta formulada en este documento, debe ser complementada con instancias de auto y coevaluación, para que adquiera una dimensión integral, lo que permitirá hacer del diseño de evaluación de aprendizajes significativos, una herramienta curricular sólida que posibilitará no sólo centrar la mirada en la evaluación de resultados, sino que también de procesos y de los diagnósticos correspondientes. En esta misma 
línea, surge entonces una tarea a asegurar en los liderazgos pedagógicos de los establecimientos educacionales, estos no sólo deben supervisar las planificaciones curriculares, sino que ha de centrar la mirada en el quehacer evaluativo del docente, mediante un acompañamiento sistemático en el aula.

Por último, es gravitante hacer esfuerzos por generar una nueva y contextualizada cultura evaluativa, la que ha de estar focalizada en la mejora permanente de los aprendizajes, conceptuales y proposicionales preferentemente. Para ello es urgente abordar algunas complejidades, que en lenguaje de Salazar (2013a y 2012) se conocen como nudos de la evaluación educativa. Estos se traducen en un total de quince nudos, siendo los siguientes: 1.- La tarea contradictoria, educar en valores y preparar para la vida; 2.- La multifuncionalidad docente, más tareas en los mismos tiempos; 3.- La homogenización del espacio educativo; 4.- La atención a la diversidad y el tratamiento evaluativo; 5.- El peso específico del paradigma educativo, procesos $\mathrm{v} / \mathrm{s}$ producto; 6 . - El reforzamiento privado y su impacto en la calidad de los aprendizajes; 7.- El compromiso estudiantil, requisito en los ambientes de aprendizaje; 8.- La justicia evaluativa y las exigencias académicas; 9.- La coherencia entre el aprendizaje la actividad y la evaluación; 10:- La cultura de la calificación; 11.- El uso de los resultados en la evaluación; 12.- La metaevaluación; 13.- La alianza estratégica, familia - escuela; 14.- La dimensión ética de la evaluación, rol de estudiantes y docentes; y 15.- Los instrumentos evaluativos y sus dinámicas, la precisión de los mismos y el pudor estudiantil.

"La naturaleza benigna provee de manera que en cualquier parte halles algo que aprender" (Leonardo Da Vinci).

\section{REFERENCIAS BIBLIOGRÁFICAS}

Ahumada, P. (2005). Hacia una evaluación auténtica del aprendizaje. Ciudad de México D.F.: Paidós Educador.

Ahumada, P. (2001). La evaluación en una concepción de aprendizajı significativo. Valparaíso: Universidad Católica de Valparaíso.

Alfageme, M. y Millares, P. (2009). Instrumentos de evaluación para centra nuestra ense-anza en el aprendizaje. En IBER. Didáctica de la: Ciencias Sociales Geografía e Historia. № 60. A-o XIV. Pp. 8-20.

Alonso, C; Gallego, D. y Honey, P. (2007). Los estilos de aprendizaje procedimientos de diagnóstico y mejora. ( $7^{\underline{a}}$ Ed.). Bilbao: Mensajero.

Ausubel, D. (1963). The psychology of meaningful verbal learning. New York Grune \& Stratton. 
Ausubel, D. (1968). Educational psychology: a cognitive view. New York: Holt, Rinehart, and Winston. 685 pp https://doi.org/10.1080/00461526809528961 https://doi.org/10.1037/h0025943

Ausubel, D. (1976). Psicología Educativa. México: Trillas.

Ausubel, D.; Novak, J. \& Hanesian, H. (1991). Psicología educativa: un punto de vista cognitivo. 5a Ed. Ciudad de México: Trillas.

Barberá, E. (1999). Evaluación de la enseñanza, evaluación del aprendizaje. Barcelona: EDEBÉ.

Bonvecchio M. y Maggioni B. (2006). Evaluación de los aprendizajes. Manual para docentes. Buenos Aires: Novedades Educativas.

Castillo, S, y Cabrerizo, J. (2003a). Evaluación Educativa y Promoción Escolar. Madrid: Pearson.

Castillo, S. y Cabrerizo, J. (2003b). Prácticas de evaluación educativa. Madrid: Pearson.

Coll, C. y Martín, E. (1993). La evaluación del aprendizaje en el curriculum escolar: una perspectiva constructivista. En Coll, Martín, Mauri, Miras, Onrubia, Solé y Zabalza. El Constructivismo en el aula. Barcelona: GRAO.

Díaz-Barriga, F. y Hernández, G. (2005). Estrategias docentes para un aprendizaje significativo: Una interpretación constructivista. ( $2^{\mathrm{a}} \mathrm{Ed}$.) Ciudad de México D.F.: Mcgraw-hill.

Marchesi, A. y Martín, E. (1998). Calidad de la enseñanza en tiempos de cambio. Madrid: Alianza.

MINISTERIO DE EDUCACIÓN. (2006). Evaluación para el aprendizaje. Enfoque y materiales prácticos para lograr que sus estudiantes aprendan más y mejor. Santiago: Unidad de currículum y evaluación.

Moreira, M. (2000). Aprendizaje significativo: teoría y práctica. Madrid: Visor.

Novak, J. y Gowin, D. (1988). Aprendiendo a aprender. Barcelona: Martínez Roca.

Ontoria, A. (1993). Mapas conceptuales. Una técnica para aprender. Madrid: Narcea. 
Román, M. (1999). Aprendizaje y currículum: Didáctica socio - cognitiva aplicada. Madrid: Eos.

Salazar, J. (2013a). Evaluación educativa y estilos de aprendizaje. En: García, J; Jiménez, M; Martínez, T, y Sánchez C. (Coord.). Estilos de aprendizaje y otras perspectivas pedagógicas del Siglo XXI. (165 185). Texcoco. Colegio de Postgraduados en Ciencias Agrícolas.

Salazar, J. (2013b). Evaluación de aprendizajes significativos en jóvenes y adultos. En: Williamson, G; Salazar, J; González, J; y Pérez, I. (Eds.). Educación social y educación de jóvenes y adultos. Reflexiones, experiencias y propuestas. $(89-95)$. Temuco. Ediciones Universidad de La Frontera.

Salazar, J. (2012). Los nudos de la evaluación docente: La autoevaluación y su espacio de reflexión pedagógica. Conferencia de Clausura en el IV Congreso Internacional de Evaluación de la Función Docente. Universidad de La Frontera (Chile). 27 y 28 de abril.

Salazar, J. (2009). Desafíos de la evaluación en la educación virtual. Conferencia presentada en las II Jornadas Internacionales de Tecnologías Aplicadas a la Enseñanza de las Lenguas. Universidad Nacional de Córdoba (Argentina). 27 al 29 de Mayo.

Salazar, J. (2007). Evaluación de aprendizajes en ambientes virtuales: una aproximación al sector de lenguas y comunicación. Conferencia presentada en las Primeras Jornadas Internacionales de Nuevas Tecnologías Aplicadas a la Enseñanza de Lenguas (Materna y/o Extranjera(s)). Universidad Nacional de Córdoba (Argentina). 21 al 23 de Mayo.

Salazar, J. e Isla, R. (2003). El accionar del trabajo cooperativo: una propuesta en el contexto de una evaluación alternativa, auténtica y de desempeño. Novedades Educativas. № 150. A-o 15. 36-39.

Salazar, J., Isla, R. y Muñoz, R. (2002). Significado de las notas y rol de los padres. Novedades Educativas. № 135. A-o 14. 12 -13

Salazar, J. y Marques, M. (2012). Acompañamiento al aula: una estrategia para el mejoramiento del trabajo pedagógico. En: Revista lberoamericana de Evaluación Educativa. V.5, № 1 (e). 10 - 20.

Shuell, T. (1990). Phases of meaningful learning. Review of educational research, $60,4.631$ - 548. https://doi.org/10.3102/00346543060004531 
Solé, I. y Coll, C. (1993). Los profesores y la concepción constructivista. En C. Coll, E. Martín, T. Mauri, M. Miras, J. Onrubia, e I. Solé (Eds.), El constructivismo en el aula (pp. 7-23). Barcelona, España: Graó.

Tyler, R. (1942). General Statement on Evaluation. The Journal of Educational Research, 35(7), 492-501. https://doi.org/10.1080/00220671.1942.10881106 